\title{
Quasi-Newton Methods and their Application to Function Minimisation
}

\author{
By C. G. Broyden
}

1. Introduction. The solution of a set of $n$ nonlinear simultaneous equations, which may be written

$$
f_{j}\left(x_{1}, x_{2}, \cdots, x_{n}\right)=0, \quad j=1,2, \cdots, n,
$$

can in general only be found by an iterative process in which successively better, in some sense, approximations to the solution are computed. Of the methods available most rely on evaluating at each stage of the calculation a set of residuals and from these obtaining a correction to each element of the approximate solution. The most common way of doing this is to take each correction to be a suitable linear combination of the residuals. There is, of course, no reason in principle why more elaborate schemes should not be used but they are difficult both to analyse theoretically and to implement in practice.

The minimisation of a function of $n$ variables, for which it is possible to obtain analytic expressions for the $n$ first partial derivatives, is a particular example of this type of problem. Any technique used to solve nonlinear equations may be applied to the expressions for the partial derivatives but, because it is known in this case that the residuals form the gradient of some function, it is possible to introduce refinements into the method of solution to take account of this extra information. Since, in addition, the value of the function itself is known, further refinements are possible.

The best-known method of solving a general set of simultaneous nonlinear equations, in which the corrections are computed as linear combinations of the residuals, is the Newton-Raphson method. The principal disadvantage of this method lies in the necessity of evaluating and inverting the Jacobian matrix at each stage of the iteration and so a number of methods have arisen, e.g. [1], [2], [4] and [8] in which the inverse Jacobian matrix is replaced by an approximation which is modified in some simple manner at each iteration. Although each method has its own peculiarities certain properties are common to a large class of these methods, and several of these are discussed here. In particular, if it is known that the functions to be zeroed are the first partial derivatives of a function $F$, then it is possible, if $F$ is quadratic, to modify the approximating matrix in such a way that $F$ is minimised in a finite number of steps. This method of modification is not unique and leads to a subclass of methods of which one example is the method of Davidon [3] as amended by Fletcher and Powell [4].

Since in the methods under discussion the corrections are computed as linear combinations of the residuals, it is natural to introduce matrix notation. Thus a function $f_{j}$ of the variables $x_{1}, x_{2}, \cdots, x_{n}$, may be regarded as a function of the $n$th order vector $\mathbf{x}$, and each $f_{j}$ in turn may be treated as the $j$ th element of the $n$th

Received March 24, 1966. Revised October 25, 1966. 
order vector $\mathrm{f}$. In this notation the basic problem becomes that of finding a vector $\mathbf{x}$ such that

$$
f(x)=0 \text {. }
$$

If the $i$ th approximation to the solution of (1.2) is denoted by $\mathbf{x}_{i}$ the notation may be simplified by referring to $\mathbf{f}\left(\mathbf{x}_{i}\right)$ as $\mathbf{f}_{i}$. This vector is the vector of residuals at the $i$ th stage of the process, and since the correction to $\mathbf{x}_{i}$ is to be given as a linear combination of the residuals it follows that the process may be defined by

$$
\begin{gathered}
\mathbf{p}_{i}=-\mathbf{H}_{i} \mathbf{f}_{i}, \\
\mathbf{x}_{i+1}=\mathbf{x}_{i}+\mathbf{p}_{i} t_{i},
\end{gathered}
$$

where $\mathbf{H}_{i}$ and $t_{i}$ are an $n$th order matrix and a scalar respectively. It is clear from these equations that the inclusion of $t_{i}$ is not strictly necessary as it could well be absorbed in the matrix $\mathbf{H}_{i}$. It is convenient to treat it as a separate entity, however, since in practice its value is often not known until after the correction vector $\mathbf{p}_{i}$ has been computed.

The class of algorithms defined by (1.3) and (1.4) is extremely general. If, for instance, the functions $f_{j}$ are the first partial derivatives of a function $F, \mathbf{H}_{i}$ is the unit matrix, and $t_{i}$ is chosen to minimise $F$, then (1.3) and (1.4) define the classical method of steepest descent. If $\mathbf{H}_{i}$ is $\mathbf{A}_{i}^{-1}$, where $\mathbf{A}_{i}$ is the Jacobian matrix evaluated at $\mathbf{x}_{\imath}$, and $t_{i}$ is unity then the method defined by the algorithm becomes that of Newton. Finally, $\mathbf{H}_{i}$ may be chosen to satisfy certain conditions discussed more fully in Section 2 below. Since these conditions ensure that some properties of $\mathbf{H}_{i}$ approximate to those of $\mathbf{A}_{i}^{-1}$ these methods may be regarded as variations of Newton's method. For this reason, and for brevity, they will be referred to in the subsequent discussion as quasi-Newton methods.

2. Quasi-Newton Methods. The quasi-Newton methods may be defined as those methods for which the iteration matrix $\mathbf{H}_{i+1}$ satisfies the equation

$$
\mathbf{H}_{i+1} \mathbf{y}_{i}=\mathbf{p}_{i} s_{i}
$$

where

$$
\mathbf{y}_{i}=\mathbf{f}_{i+1}-\mathbf{f}\left(\mathbf{x}_{i+1}-\mathbf{p}_{i} s_{i}\right)
$$

$\mathbf{p}_{i}$ is identified with the $\mathbf{p}_{i}$ of (1.3) and (1.4) and $s_{i}$ is some scalar whose choice is discussed subsequently.

Assume now that $\mathrm{f}$ is differentiable at $\mathbf{x}_{i+1}$. It then follows that (see e.g. [6], pp. 188-189)

$$
\mathbf{f}\left(\mathbf{x}_{i+\mathbf{1}}-\mathbf{p}_{i} s_{i}\right)=\mathbf{f}_{i+\mathbf{1}}-\mathbf{A}_{i+\mathbf{1}} \mathbf{p}_{i} s_{i}+\mathbf{r}\left(\mathbf{p}_{i} s_{i}\right),
$$

where $\mathbf{A}_{i+\mathbf{1}}$ is the Jacobian matrix evaluated at $\mathbf{x}_{i+\mathbf{1}}$ and where $\mathbf{r}\left(\mathbf{p}_{i} s_{i}\right)$ is small in the sense that

$$
\lim _{\left\|\mathbf{p}_{i} s_{i}\right\| \rightarrow 0} \frac{\left\|\mathbf{r}\left(\mathbf{p}_{i} s_{i}\right)\right\|}{\left\|\mathbf{p}_{i} s_{i}\right\|}=0
$$

the vertical bars indicating the Euclidean norm. Hence 


$$
\mathbf{y}_{i}=\mathbf{A}_{i+1} \mathbf{p}_{i} s_{i}-\mathbf{r}\left(\mathbf{p}_{i} s_{i}\right)
$$

so that, if $\mathbf{A}_{i+1}$ is nonsingular, Eqs. (2.1) and (2.4) give

$$
\mathbf{H}_{i+1} \mathbf{y}_{i}=\mathbf{A}_{i+1}^{-1}\left(\mathbf{y}_{i}+\mathbf{r}\left(\mathbf{p}_{i} s_{i}\right)\right) \text {. }
$$

Thus if $s_{i}\left\|\mathbf{p}_{i}\right\| \rightarrow 0$, as it does when $x_{i}$ approaches the solution since then $\left\|\mathbf{p}_{i}\right\| \rightarrow 0$, it follows that

$$
\mathbf{H}_{i+1} \mathbf{y}_{i} \rightarrow \mathbf{A}_{i+1}^{-1} \mathbf{y}_{i}
$$

so that $\mathbf{H}_{i+1}$ will have at least one property of the inverse Jacobian matrix, justifying to some extent the use of the term "quasi-Newton".

A somewhat more lengthy discussion of the derivation of (2.1) and its identification with Newton's method is given by Broyden [2]. In that discussion, however, the matrix $\mathbf{H}_{i}$ is defined to be the negative of the $\mathbf{H}_{i}$ defined here. The definition of $\mathbf{H}_{i}$ in the present work follows that of Fletcher and Powell, and is more nearly consistent with the usage of Traub [7] than was the definition in [2].

In the examples already published of this class of methods $s_{i}$ has nearly always been taken to be $t_{i}$, and it will be so chosen in what follows. With this value of $s_{i}$, (2.1) and (2.2) become, from (1.4),

$$
\begin{aligned}
\mathrm{H}_{i+1} \mathrm{y}_{i} & =\mathrm{p}_{i} t_{i}, \\
\mathrm{y}_{i} & =\mathrm{f}_{i+1}-\mathrm{f}_{i} .
\end{aligned}
$$

Eq. (2.6), although a little less general than (2.1), is still not sufficient to define $\mathbf{H}_{i+1}$ or even to give any indication of how it may be derived. Since, however, a matrix $\mathbf{H}_{i}$ which possesses to some extent the properties of the inverse Jacobian matrix is already available it would appear reasonable to obtain $\mathbf{H}_{i+1}$ by adding some correction to $\mathbf{H}_{i}$. Denote this correction by $\mathbf{B}_{i}$. Then

$$
\mathbf{H}_{i+1}=\mathbf{H}_{i}+\mathbf{B}_{i}
$$

and, from (2.6),

$$
\mathbf{B}_{i} \mathbf{y}_{i}=\mathbf{p}_{i} t_{i}-\mathbf{H}_{i} \mathbf{y}_{i} .
$$

The simplest (in the sense of least rank) matrix that $\mathbf{B}_{i}$ can be so that (2.8) is satisfied is

$$
\mathbf{B}_{i}=\left(\mathbf{p}_{i} t_{i}-\mathbf{H}_{i} \mathbf{y}_{i}\right) \mathbf{z}_{i}{ }^{T}
$$

where $\mathbf{z}_{i}$ is arbitrary except for the normalisation condition that

$$
\mathbf{z}_{i}{ }^{T} \mathbf{y}_{i}=1
$$

This, however, is a little too restrictive and a more general alternative is

$$
\mathbf{B}_{i}=\mathbf{p}_{i} t_{i} \mathbf{q}^{T}-\mathbf{H}_{i} \mathbf{y}_{i} \mathbf{z}_{i}{ }^{T}
$$

where both $\mathbf{q}_{i}$ and $\mathbf{z}_{i}$ are arbitrary but subject again to the condition that

$$
\mathrm{q}_{i}{ }^{T} \mathrm{y}_{i}=\mathbf{z}_{i}{ }^{T} \mathbf{y}_{i}=1 \text {. }
$$

The remainder of this work is devoted to the discussion of methods where $\mathbf{B}_{i}$ is given by $\mathrm{Eq}$. (2.10), so that $\mathbf{H}_{i+1}$ is given by 


$$
\mathrm{H}_{i+1}=\mathrm{H}_{i}-\mathrm{H}_{i} \mathbf{y}_{i} \mathbf{z}_{i}{ }^{T}+\mathbf{p}_{i} t_{i} \mathbf{q}_{i}{ }^{T} .
$$

This equation includes as special cases the generalised secant method of solving nonlinear simultaneous equations [1], [5], [7] and [8], a method due to Broyden of solving nonlinear simultaneous equations [2], the modified Davidon method of function minimisation [4], and the generalisation of Davidon's method, discussed below, of function minimisation.

3. Stability. A necessary condition that the algorithm defined by (1.3), (1.4) and (2.11) will solve an arbitrary minimisation problem is that at no stage of the process, i.e., for no $i$, will either $\mathbf{q}_{i}$ or $\mathbf{z}_{i}$ be orthogonal to $\mathbf{y}_{i}$, since it then becomes impossible to satisfy (2.10b). Another possible cause of the failure of the algorithm to converge is the singularity of some $\mathbf{H}_{i}$, and as a first step in investigating why this should be so the following lemma is proved.

LemmA. For an algorithm defined by (1.3), (1.4) and (2.11), $\mathbf{x}_{i+r}$, where $r \geq 1$, is given by

$$
\mathbf{x}_{i+r}=\mathbf{x}_{i}-\mathbf{H}_{i} \mathbf{v}_{i r}
$$

where $\mathbf{v}_{\text {ir }}$ is some vector.

Proof. The proof is inductive. It follows from (1.3) and (2.11) that, if $\mathbf{M}_{i}$ is defined by

$$
\mathbf{M}_{i}=\left(\mathbf{I}-\mathbf{y}_{i} \mathbf{z}_{i}{ }^{T}-\mathbf{f}_{i} t_{i} \mathbf{q}_{i}{ }^{T}\right),
$$

then

$$
\mathbf{H}_{i+1}=\mathbf{H}_{i} \mathbf{M}_{i} \text {. }
$$

Hence

$$
\mathbf{H}_{i+r}=\mathbf{H}_{i} \mathbf{M}_{i} \mathbf{M}_{i+1} \cdots \mathbf{M}_{i+r-1} .
$$

Assume now that $\mathbf{x}_{i+r}$ is given by (3.1). Then from (1.3) and (1.4)

$$
\begin{aligned}
\mathbf{x}_{i+r+1} & =\mathbf{x}_{i}-\mathbf{H}_{i} \mathbf{v}_{i r}-\mathbf{H}_{i+r} \mathbf{f}_{i+r} t_{i+r}, \\
& =\mathbf{x}_{i}-\mathbf{H}_{i}\left(\mathbf{v}_{i r}+\mathbf{M}_{i} \mathbf{M}_{i+1} \cdots \mathbf{M}_{i+r-1} \mathbf{f}_{i+r} t_{i+r}\right), \\
& =\mathbf{x}_{i}-\mathbf{H}_{i} \mathbf{v}_{i, r+1} .
\end{aligned}
$$

Since, from (1.3) and (1.4), (3.1) holds for $r=1$, it holds for all $r \geq 1$, and this proves the lemma. Now if the algorithm is to converge to a solution $\mathbf{x}$,

$$
\lim _{r \rightarrow \infty} \mathbf{x}_{i+r}=\mathbf{x}
$$

and it follows from (3.1) that

$$
\lim _{r \rightarrow \infty} \mathbf{H}_{i} \boldsymbol{v}_{i r}=\mathbf{x}_{i}-\mathbf{x} .
$$

Now if $\mathbf{H}_{i}$ is nonsingular it is always possible that (3.3) will hold, but if not it will hold only if the vector $\mathbf{x}_{i}-\mathbf{x}$ satisfies certain conditions. Since these conditions in general will not be fulfilled, it is desirable that during the course of the algorithm $\mathbf{H}_{i}$ should never become singular. Quasi-Newton algorithms for which $\mathbf{q}_{i}{ }^{T} \mathbf{y}_{i}$ and 
$\mathbf{z}_{i}{ }^{T} \mathbf{y}_{i}$ never become zero and $\mathbf{H}_{i}$ never becomes singular will be regarded as stable, and it is shown subsequently that the modified Davidon method and its generalisation are both stable according to this definition.

It should perhaps be emphasised here that stability as defined above is not sufficient to guarantee that a particular algorithm will converge. For, consider methods 1 and 2 for solving nonlinear simultaneous equations, defined and discussed by Broyden, [2]. Of these, the second is stable and the first is not, since in the first method it is possible that $\mathbf{z}_{i}^{T} \mathbf{y}_{i}=0$. However, in practice, the first method solves equations and the second is unable to do so. Neither is it correct to infer that an algorithm for function minimisation will converge to the solution merely because the function is reduced at each step, for the sequence of function values $F_{i}$ obtained by the algorithm may well have a lower bound that is greater than the minimum value of $F$ even for $F$ strictly convex. The most that can be inferred from the fact that $F_{i+1}<F_{i}$ is that the algorithm cannot diverge, a somewhat tautologous conclusion.

4. Linear Systems. If a quasi-Newton method is to solve effectively a general set of nonlinear equations intuition would suggest that it would solve a general set of linear equations in a finite number of steps, and indeed some investigators set great store by this property. Although the present author is not convinced of its desirability, it is relevant to examine sufficient conditions for its attainment, since they do suggest the form that particular algorithms might take.

Let then $k$ and $s$ be two positive integers and define the matrices $\mathbf{Y}_{k, s}, \mathbf{Z}_{k, s}$, $\mathbf{B}_{k, s}, \mathbf{P}_{k, s}, \mathbf{Q}_{k, s}, \mathbf{T}_{k, s}$ and $\mathbf{G}_{k, s}$ as follows, where all the vectors concerned are of order $n$.

$$
\begin{aligned}
& \mathbf{Y}_{k, s}=\left[\mathbf{y}_{k}, \mathbf{y}_{k+1}, \cdots, \mathbf{y}_{k+s-1}\right] \\
& \mathbf{Z}_{k, s}=\left[\mathbf{z}_{k}, \mathbf{z}_{k+1}, \cdots, \mathbf{z}_{k+s-1}\right] \\
& \mathbf{B}_{k, s}=\left(\mathbf{I}-\mathbf{y}_{k} \mathbf{z}_{k}^{T}\right)\left(\mathbf{I}-\mathbf{y}_{k+1} \mathbf{z}_{k+1}^{T}\right) \cdots\left(\mathbf{I}-\mathbf{y}_{k+s-1} \mathbf{z}_{k+s-1}^{T}\right) \\
& \mathbf{P}_{k, s}=\left[\mathbf{p}_{k}, \mathbf{p}_{k+1}, \cdots, \mathbf{p}_{k+s-1}\right] \\
& \mathbf{Q}_{k, s}=\left[\mathbf{q}_{k}, \mathbf{q}_{k+1}, \cdots, \mathbf{q}_{k+s-1}\right] \\
& \mathbf{T}_{k, s}=\operatorname{diag}\left(t_{k}, t_{k+1}, \cdots, t_{k+s-1}\right) \\
& \mathbf{G}_{k, s}=\left[\mathbf{B}_{k+1, s-1}^{T} \mathbf{q}_{k}, \mathbf{B}_{k+2, s-2}^{T} \mathbf{q}_{k+1}, \cdots, \mathbf{B}_{k+s-1,1}^{T} \mathbf{q}_{k+s-2}, \mathbf{q}_{k+s-1}\right]
\end{aligned}
$$

Then, from Eq. (2.11) with $i=k, k+1, \cdots, k+s-1$ and the above definitions it follows that

$$
\mathbf{H}_{k+8}=\mathbf{H}_{k} \mathbf{B}_{k, s}+\mathbf{P}_{k, 8} \mathbf{T}_{k, s} \mathbf{G}_{k, 8}^{T} .
$$

Consider now Eq. (4.8). The first term on the right-hand side consists of $\mathbf{H}_{k}$ modified by post-multiplication by $\mathbf{B}_{k, s}$ and the second term consists solely of information derived from the last $s$ steps of the process or injected during these steps by the choice of $\mathrm{q}_{i}$ and $\mathrm{z}_{i}, i=k, k+1, \cdots, k+s-1$. Now it is reasonable to require of $\mathbf{H}_{k+s}$ that it should comprise the latest information derived from the iteration so that the first term on the right-hand side of (4.8), which represents essentially old information, should tend to the null matrix as $s$ increases. Since $\mathbf{H}_{k}$ 
is nonsingular, this is achieved if and only if $\mathbf{B}_{k, s}$ tends to the null matrix as $s \rightarrow \infty$. A more stringent requirement is that $\mathbf{B}_{k, s}$ becomes the null matrix after a finite number of steps. If this occurs, then $\mathbf{H}_{k+s}$ will comprise information derived only from a finite number of the most recent steps, old information having been completely purged. It will now be shown that $\mathbf{B}_{k, s}$ cannot be null for $s<n$, and necessary and sufficient conditions for its nullity will be established.

THEOREM 1. If $\mathbf{Y}_{k, n}, \mathbf{Z}_{k, n}$ and $\mathbf{B}_{k, n}$ are as defined by Eqs. (4.1)-(4.3) then the necessary and sufficient condition for $\mathbf{B}_{k, n}$ to be null is that the product $\mathbf{Z}_{k, n}^{T} \mathbf{Y}_{k, n}$ is unit upper triangular, i.e., that

$$
\begin{array}{ll}
\mathbf{z}_{i}{ }^{T} \mathbf{y}_{i}=1, & i=k, k+1, \cdots, k+n-1, \\
\mathbf{z}_{i}{ }^{T} \mathbf{y}_{j}=0, & k \leq j<i \leq k+n-1 .
\end{array}
$$

Proof. (a) Sufficiency. Since both $\mathbf{Y}_{k, n}$ and $\boldsymbol{Z}_{k, n}$ are square, the condition that the product $\boldsymbol{Z}_{k, n}^{\boldsymbol{T}} \mathbf{Y}_{k, n}$ is unit upper triangular implies that $\mathbf{Y}_{k, n}$ is nonsingular. From (4.3) and (4.9) it may be verified directly that $\mathbf{B}_{k, n} \mathbf{y}_{i}=0, i=k, k+1, \cdots$, $k+n-1$, so that $\mathbf{B}_{k, n} \mathbf{Y}_{k, n}=\mathbf{0}$, and the result follows from the nonsingularity of $\mathbf{Y}_{k, n}$.

(b) Necessity. Direct expansion of the right-hand side of (4.3) gives, from the postulated nullity of $\mathbf{B}_{k, n}$,

$$
\mathbf{0}=\mathbf{I}-\mathbf{Y}_{k, n} \mathbf{V}_{n} \mathbf{Z}_{k, n}^{T},
$$

where $\mathbf{V}_{n}$ is some $n \times n$ unit upper triangular matrix. Since, from (4.10), $\mathbf{Y}_{k, n}$ cannot be singular, it follows that

$$
\mathbf{I}=\mathbf{V}_{n} \mathbf{Z}_{k, n}^{T} \mathbf{Y}_{k, n}
$$

and since the inverse of the unit upper triangular matrix $\mathrm{V}_{n}$ is itself unit upper triangular, the result follows.

Corollary. $\mathbf{B}_{k, s}$ cannot be null for $s<n$, since then a vector $\mathbf{w}$ could aluays be found such that $\mathbf{w}^{T} \mathbf{Y}_{k, s}=\mathbf{0}$, so that, since

$$
\mathbf{B}_{k, s}=\mathbf{I}-\mathbf{Y}_{k, s} \mathbf{V}_{s} \mathbf{Z}_{k, s}^{T}, \quad \mathbf{w}^{T} \mathbf{B}_{k, s}=\mathbf{w}^{T} .
$$

A quasi-Newton method that solves a set of linear equations in a finite number of steps will be said to be exact, and we now show that the algorithm about to be defined is exact according to this definition. Let $\mathbf{H}_{k}$ be arbitrary but nonsingular and $\mathbf{x}_{k}$ be arbitrary, and let

$$
\begin{aligned}
& \mathbf{p}_{i}=-\mathbf{H}_{i} \mathbf{f}_{i}, \\
& \mathbf{x}_{i+1}=\mathbf{x}_{i}+\mathbf{p}_{i} t_{i}, \\
& \mathbf{y}_{i}=\mathbf{f}_{i+1}-\mathbf{f}_{i}, \\
& \text { (4.11d) } \quad \mathbf{H}_{i+1}=\mathbf{H}_{i}-\mathbf{H}_{i} \mathbf{y}_{i} \mathbf{z}_{i}{ }^{T}+\mathbf{p}_{i} t_{i} \mathbf{q}_{i}{ }^{T}
\end{aligned}
$$

$$
i=k, k+1, \cdots, k+r-1,
$$

where $t_{i}$ is arbitrary but nonzero and the otherwise arbitrary vectors $\mathbf{z}_{i}$ and $\mathbf{q}_{i}$ satisfy

$$
\begin{array}{ll}
\mathbf{z}_{i}{ }^{T} \mathbf{y}_{i}=1, & i=k, k+1, \cdots, k+r-1, \\
\mathbf{z}_{i}{ }^{T} \mathbf{y}_{j}=0, & k \leq j<i \leq k+r-1,
\end{array}
$$




$$
\begin{array}{ll}
\mathbf{q}_{i}{ }^{T} \mathbf{y}_{i}=1, & i=k, k+1, \cdots, k+r-1, \\
\mathbf{q}_{i}{ }^{T} \mathbf{y}_{j}=0, & k \leq j<i \leq k+r-1,
\end{array}
$$

where $1 \leq r \leq n$.

Theorem 2. If the algorithm defined by Eqs. (4.11) is applied to the linear function

$$
\mathbf{f}=\mathbf{A x}-\mathbf{b}
$$

where $\mathbf{A}$ is an $n \times n$ matrix and $\mathbf{b}$ an nth-order vector then, with $\mathbf{Y}_{k, s}$ as defined by Eq. (4.1),

$$
\left(\mathbf{A H}_{k+s}-\mathbf{I}\right) \mathbf{Y}_{k, s}=\mathbf{0}, \quad \mathbf{1} \leq s \leq r, 1 \leq r \leq n .
$$

Proof. It follows from Eqs. (4.1), (4.3) and (4.11e, f) that

$$
\begin{aligned}
\mathbf{B}_{k, j} \mathbf{Y}_{k, j} & =\mathbf{0}, \\
\mathbf{B}_{k+j, s-j} \mathbf{Y}_{k, j} & =\mathbf{Y}_{k, j}, \quad 1 \leq j<s, 1<s \leq r, \\
\mathbf{B}_{k+j, s-j} \mathbf{Y}_{k+j, s-j} & =\mathbf{0},
\end{aligned}
$$

so that, from Eqs. (4.7), (4.11g, h) and (4.13),

$$
\mathbf{G}_{k, s}^{T} \mathbf{Y}_{k, s}=\mathbf{I}, \quad 1 \leq s \leq r
$$

where the unit matrix is of order $s$. Thus, from (4.8), (4.13a) and (4.14),

$$
\mathbf{H}_{k+s} \mathbf{Y}_{k, s}=\mathbf{P}_{k, s} \mathbf{T}_{k, s}, \quad 1 \leq s \leq r .
$$

Now for the linear system (4.14) it is easy to verify that

$$
\mathbf{A P}_{k, s} \mathbf{T}_{k, s}=\mathbf{Y}_{k, s}
$$

so that, from (4.15),

$$
\left(\mathbf{A H}_{k+s}-\mathbf{I}\right) \mathbf{Y}_{k, s}=0, \quad 1 \leq s \leq r, 1 \leq r \leq n,
$$

and the theorem is proved.

Corollary. Eqs. (4.11e, f, g, h) can be satisfied for $r=n$ only if $\mathbf{A}$ is nonsingular since they imply that $\mathbf{Y}_{k, n}$ is nonsingular, and hence, from Eq. (4.17), that

$$
\mathbf{A H}_{k+n}=\mathbf{I} \text {. }
$$

Thus not only is $\mathbf{A}$ nonsingular but $\mathbf{H}_{k+n}=\mathbf{A}^{-1}$, and the algorithm is thus exact, since a further step must lead to the correct solution.

We now consider some existing algorithms in the light of the two theorems. The only quasi-Newton method for which $(4.11 \mathrm{e}, \mathrm{f}, \mathrm{g}, \mathrm{h})$ hold for $r=n$ and all $k$ is the generalised secant method, [1], [5], [7], [8]. It follows from Theorem 1 that $\mathbf{B}_{k+n}$ is null for all $k$, so that (4.8) becomes

$$
\mathbf{H}_{k+n}=\mathbf{P}_{k, n} \mathbf{T}_{k, n} \mathbf{G}_{k, n}^{T}
$$

and this, from (4.14), may be written

$$
\mathbf{H}_{k+n}=\mathbf{P}_{k, n} \mathbf{T}_{k, n} \mathbf{Y}_{k, n}^{-1}, \quad k \geq 1 .
$$

Eq. (4.18), which is frequently taken as defining the generalised secant method, does not specify $\mathbf{H}_{i}, 1 \leq i \leq n$, and Barnes [1] suggests a possible way of doing 
this. A minor disadvantage of the method is that every step requires the solution of a set of linear equations, although this may be overcome at the cost of storing an additional $n \times n$ matrix. A more serious disadvantage is that, without some modification, it does not appear to work. Although it is an exact method it tends to be unstable for strongly nonlinear problems, since either $\mathbf{Y}_{k, n}$ or $\mathbf{P}_{k, n}$ may be effectively singular for some $k$. Then either $\mathbf{H}_{k+n}$ is not defined, or it becomes singular giving rise to subsequent convergence problems as discussed in Section 3 above. Barnes gives a method of overcoming this difficulty but at the cost of complicating the algorithm.

In Broyden's method [2] for solving nonlinear simultaneous equations the vectors $\mathbf{q}_{i}{ }^{T}$ and $\mathbf{z}_{i}{ }^{T}$ are given by

$$
\mathbf{q}_{i}{ }^{T}=\mathbf{z}_{i}{ }^{T}=\left(\mathbf{p}_{i}{ }^{T} \mathbf{H}_{i} \mathbf{y}_{i}\right)^{-1} \mathbf{p}_{i}{ }^{T} \mathbf{H}_{i} .
$$

The method is not exact, although when applied to the linear function (4.12) it can be shown that

$$
\left\|\mathbf{H}_{i+1}^{-1}-\mathbf{A}\right\| \leq\left\|\mathbf{H}_{i}^{-1}-\mathbf{A}\right\|
$$

for the spectral norms of the matrices concerned. Neither is the method stable for although $\mathrm{H}_{i}$ can become singular only after a zero step, it is theoretically possible, with the choice of $t_{i}$ given in [2], that $\mathbf{p}_{i}{ }^{T} \mathbf{H}_{i} \mathbf{y}_{i}=0$. This contingency never occurred when solving the admittedly limited number of problems described in [2] and could be overcome, if necessary, merely by changing the somewhat permissive criterion for determining $t_{i}$.

If $\mathbf{q}_{i}{ }^{T}$ and $\mathbf{z}_{i}{ }^{T}$ are given by

$$
\begin{aligned}
& \mathbf{q}_{i}{ }^{T}=\left(\mathbf{p}_{i}{ }^{T} \mathbf{y}_{i}\right)^{-1} \mathbf{p}_{i}{ }^{T}, \\
& \mathbf{z}_{i}{ }^{T}=\left(\mathbf{y}_{i}{ }^{T} \mathbf{H}_{i} \mathbf{y}_{i}\right)^{-1} \mathbf{y}_{i}{ }^{T} \mathbf{H}_{i},
\end{aligned}
$$

the algorithm becomes that of Davidon [3] as subsequently modified by Fletcher and Powell [4]. When this algorithm is applied to a nonlinear system, Eqs. (4.11f, $\mathrm{h}$ ) are not in general satisfied, but if applied to the linear system (4.12) they are satisfied provided that $\mathbf{A}$ is symmetric and each $t_{i}$ is precisely chosen. If, moreover, $\mathbf{A}$ is positive definite the algorithm is completely stable, and this feature, coupled with the fact that it is exact, makes it very suitable for function minimisation.

In Section 6 below we discuss algorithms where $\mathrm{q}_{i}{ }^{T}$ and $\mathrm{z}_{i}{ }^{T}$ are chosen in a more sophisticated manner than (4.19), and it is shown that these algorithms, of which that of Fletcher and Powell appears as a special case, are also stable and exact. Since they involve an arbitrary parameter, it is hoped that a suitable choice of this would lead to an improved algorithm for function minimisation.

5. Linear Systems with Symmetric Matrices. Although of the methods discussed here the generalised secant method is the only one that is exact for a general linear system it is possible, if the matrix concerned is symmetric, to construct a number of exact methods. In these the vectors $\mathbf{q}_{i}$ and $\mathbf{z}_{i}$ are not chosen specifically to satisfy Eqs. (4.11f, $\mathrm{h}$ ) as they are in the secant method but are chosen in such a way that these equations are satisfied automatically when the algorithm is applied to a linear system having a symmetric matrix. The algorithms are thus exact but not neces- 
sarily stable, and it is seen in Section 6 below that stability is to be associated with the positive-definiteness of the matrix concerned or, when the algorithm is used to minimise some nonquadratic function, with the convexity of this function. In this section we derive some further properties of the general algorithm (4.11) when applied to a linear system with symmetric matrix as a preliminary to the discussion of the particular algorithms in the following section. In order to simplify the notation $k$ is subsequently assumed to be unity and those matrices, e.g. $\mathbf{Y}_{k, s}$, requiring a double subscript are written with the first subscript, which is always unity, omitted, i.e., $\mathbf{Y}_{s}$.

Theorem 3. If the algorithm defined by Eqs. (4.11) is applied to the linear function

$$
\mathbf{f}=\mathbf{A x}-\mathbf{b},
$$

where $\mathbf{A}$ is symmetric, then

$$
t_{i} \mathbf{p}_{i}{ }^{T} \mathbf{y}_{j}=t_{j} \mathbf{p}_{j}{ }^{T} \mathbf{y}_{i}
$$

for $1 \leq i \leq r$ and all $j$.

Proof. From Theorem 2 and the postulated symmetry of $\mathbf{A}$ it follows that

$$
\mathbf{Y}_{s}^{T}\left(\mathbf{H}_{s+1}^{T} \mathbf{A}-\mathbf{I}\right) \mathbf{v}=0, \quad 1 \leq s \leq r,
$$

for any arbitrary vector $\mathbf{v}$. Putting $\mathbf{v}=\mathbf{p}_{j} t_{j}$ then gives, since

$$
\begin{aligned}
\mathbf{A p}_{j} t_{j} & =\mathbf{y}_{j} \text { for all } j, \\
\mathbf{Y}_{s}^{T}\left(\mathbf{H}_{s+1}^{T} \mathbf{y}_{j}-\mathbf{p}_{j} t_{j}\right) & =\mathbf{0}, \quad 1 \leq s \leq r,
\end{aligned}
$$

and this, from (4.15), becomes

$$
\mathbf{T}_{s} \mathbf{P}_{s}{ }^{T} \mathbf{y}_{j}=\mathbf{Y}_{s}{ }^{T} \mathbf{p}_{j} t_{j}, \quad 1 \leq s \leq r,
$$

proving the theorem.

Since this result is true for all $j$ it is true for $j=s+1$, and with this value of $j$ transposition of the last equation gives

$$
\mathbf{y}_{s+1}^{T} \mathbf{P}_{s} \mathbf{T}_{s}=t_{s+1} \mathbf{p}_{s+1}^{T} \mathbf{Y}_{s}, \quad 1 \leq s \leq r .
$$

TheOREM 4. If the algorithm defined by (4.11) is applied to the linear function (5.1) and both $\mathbf{A}$ and $\mathbf{H}_{s+1}$ are symmetric, $1 \leq s \leq r$, then

$$
\mathbf{p}_{s+2}^{T} \mathbf{y}_{j}=\left(\alpha_{s+1}+\beta_{s+1} t_{s+1}\right) \mathbf{p}_{s+1}^{T} \mathbf{y}_{j}, \quad 1 \leq j \leq s,
$$

where

$$
\alpha_{s+1}=1-t_{s+1}\left(1+\mathrm{q}_{s+1}^{T} \mathrm{f}_{s+1}\right)
$$

and

$$
\beta_{s+1}=\mathbf{z}_{s+1}^{T} \mathbf{f}_{s+1} .
$$

Proof. From Eqs. (1.3), (2.6), (2.7) and (2.11) it follows that

$$
\mathbf{p}_{s+2}=\mathbf{p}_{s+1} \alpha_{s+1}+\mathbf{H}_{s+1} \mathbf{y}_{s+1} \beta_{s+1} .
$$

Thus, from (5.4) and the postulated symmetry of $\mathbf{H}_{s+1}$,

$$
\mathbf{p}_{s+2}^{T} \mathbf{Y}_{s}=\alpha_{s+1} \mathbf{p}_{s+1}^{T} \mathbf{Y}_{s}+\beta_{s+1} \mathbf{y}_{s+1}^{T} \mathbf{H}_{s+1} \mathbf{Y}_{s}
$$


and this, from (4.15) and (5.3), becomes

$$
\mathbf{p}_{s+2}^{T} \mathbf{Y}_{s}=\left(\alpha_{s+1}+\beta_{s+1} t_{s+1}\right) \mathbf{p}_{s+1}^{T} \mathbf{Y}_{s}
$$

proving the theorem.

Corollary. If $\mathbf{p}_{m+1}^{T} \mathbf{y}_{m}=0$, where $1 \leq m \leq r-1$, and $\mathbf{H}_{j+1}$ is symmetric for $j=m, m+1, \cdots, r-1$, then $\mathbf{p}_{j+2}^{T} \mathbf{y}_{m}=0, j=m, m+1, \cdots, r-1$.

Proof. Repeated application of Theorem 4 with $s=m, m+1, \cdots, r-1$, and $j=m$ gives the result directly.

Theorem 5. If the algorithm defined by (4.11) is applied to the linear function (5.1), where $\mathbf{A}$ is symmetric and nonsingular, and moreover $\mathbf{H}_{s+1}$ is symmetric and $\mathbf{p}_{s+1}^{T} \mathbf{y}_{s}=0$ for $s=1,2, \cdots, r-1$, then

$$
\begin{aligned}
\mathbf{p}_{i}{ }^{T} \mathbf{y}_{j} & =0, \\
\mathbf{y}_{i}{ }^{T} \mathbf{H}_{r+1} \mathbf{y}_{j} & =0, \quad 1 \leq i \leq r, 1 \leq j \leq r, i \neq j . \\
\mathbf{y}_{i}{ }^{T} \mathbf{A}^{-1} \mathbf{y}_{j} & =0, \\
\mathbf{p}_{i}{ }^{T} \mathbf{A} \mathbf{p}_{j} & =0,
\end{aligned}
$$

Proof. From the corollary to Theorem 4 it follows that

$$
\mathbf{p}_{i}{ }^{T} \mathbf{y}_{j}=0, \quad 1 \leq j<i \leq r,
$$

and (5.5a) follows by applying Theorem 3 to this result. Now Eq. (5.5a) may be written

$$
\mathbf{P}_{r}^{T} \mathbf{Y}_{r}=\mathbf{D}_{r}
$$

where $\mathbf{D}_{r}$ is an $r$ th-order diagonal matrix, and from (4.15) this becomes

$$
\mathbf{Y}_{r}{ }^{T} \mathbf{H}_{r+1} \mathbf{Y}_{r}=\mathbf{T}_{r} \mathbf{D}_{r} \text {. }
$$

Since the product of two diagonal matrices is itself diagonal, this is equivalent to (5.5b). Now Theorem 2 gives

$$
\mathrm{H}_{r+1} \mathbf{Y}_{r}=\mathbf{A}^{-1} \mathbf{Y}_{r}
$$

and this, with (5.6), gives

$$
\mathbf{Y}_{r}{ }^{T} \mathbf{A}^{-1} \mathbf{Y}_{r}=\mathrm{T}_{r} \mathbf{D}_{r}
$$

establishing (5.5c). Now no $t_{i}$ is permitted to be zero, so that $\mathbf{T}_{r}$ cannot be singular and Eqs. (4.16) and (5.7) thus yield

$$
\mathbf{P}_{r}{ }^{T} \mathbf{A} \mathbf{P}_{r}=\mathbf{D}_{r} \mathbf{T}_{r}^{-1}
$$

establishing (5.5d) and the theorem.

6. Algorithms for Function Minimisation. If the elements of the vector $f$ are the first partial derivatives of the scalar function $F(x)$ and if they are differentiable, the Jacobian matrix is symmetric. If $F$ is in addition a quadratic function of $x$, then $f$ is given by

$$
\mathbf{f}=\mathbf{A x}-\mathbf{b}
$$

where $\mathbf{A}$ is symmetric and constant, and moreover if $F$ is strictly convex, $\mathbf{A}$ is posi- 
tive definite. The exact methods then will, provided that they are stable, minimise a quadratic function in a finite number of steps. We examine now two further ways of choosing $\mathbf{q}_{i}$ and $\mathbf{z}_{i}$ and show in each case that the algorithm obtained is exact provided that $\mathbf{A}$ is symmetric. We further show that the second algorithm is stable.

Algorithm 1.

$$
\mathbf{q}_{i}{ }^{T}=\mathbf{z}_{i}{ }^{T}=\left(\mathbf{y}_{i}{ }^{T} \mathbf{H}_{i} \mathbf{y}_{i}-t_{i} \mathbf{p}_{i}{ }^{T} \mathbf{y}_{i}\right)^{-1}\left(\mathbf{y}_{i}{ }^{T} \mathbf{H}_{i}-t_{i} \mathbf{p}_{i}{ }^{T}\right)
$$

and $t_{i}$ is arbitrary.

Algorithm 2.

$$
\begin{aligned}
& \mathbf{q}_{i}{ }^{T}=\alpha_{i} \mathbf{p}_{i}{ }^{T}-\beta_{i} \mathbf{y}_{i}{ }^{T} \mathbf{H}_{i}, \\
& \mathbf{z}_{i}{ }^{T}=\gamma_{i} \mathbf{y}_{i}{ }^{T} \mathbf{H}_{i}+\beta_{i} t_{i} \mathbf{p}_{i}{ }^{T},
\end{aligned}
$$

where

$$
\begin{aligned}
\alpha_{i} & =\left(1+\beta_{i} \mathbf{y}_{i}{ }^{T} \mathbf{H}_{i} \mathbf{y}_{i}{ }^{T}\right) / \mathbf{p}_{i}{ }^{T} \mathbf{y}_{i}, \\
\gamma_{i} & =\left(1-\beta_{i} t_{i} \mathbf{p}_{i}{ }^{T} \mathbf{y}_{i}\right) / \mathbf{y}_{i}{ }^{T} \mathbf{H}_{i} \mathbf{y}_{i},
\end{aligned}
$$

$\beta_{i}$ is arbitrary, $t_{i}$ is chosen to minimise $F$ and $\mathrm{H}_{1}$ is symmetric.

Theorem 6. Algorithm 1 is exact if $\mathbf{A}$ is symmetric and nonsingular.

Proof. The proof is by induction. Assume that

$$
\begin{array}{ll}
\mathbf{q}_{i}{ }^{T} \mathbf{y}_{i}=\mathbf{z}_{i}{ }^{T} \mathbf{y}_{i}=1, & 1 \leq i \leq r, \\
\mathbf{q}_{i}{ }^{T} \mathbf{y}_{j}=\mathbf{z}_{i}{ }^{T} \mathbf{y}_{j}=0, & 1 \leq j<i \leq r .
\end{array}
$$

It then follows from (5.2) with $s=r$ and $j=r+1$ that

$$
\left(\mathbf{y}_{r+1}^{T} \mathbf{H}_{r+1}-t_{r+1} \mathbf{p}_{r+1}^{T}\right) \mathbf{Y}_{r}=\mathbf{0}
$$

so that, if $\mathbf{q}_{r+1}$ and $\mathbf{z}_{r+1}$ are given by (6.1),

$$
\mathrm{q}_{r+1}^{T} \mathrm{Y}_{r}=\mathrm{z}_{r+1}^{T} \mathrm{Y}_{r}=\mathbf{0}
$$

and (6.3) holds with $r$ replaced by $r+1$. Now if $\mathbf{q}_{1}$ and $z_{1}$ are given by (6.1) they satisfy (4.11e, g) so that, putting $s=r=1$, (4.17) becomes

$$
\left(\mathbf{A H}_{2}-\mathbf{I}\right) \mathbf{y}_{1}=0 \text {. }
$$

Since $\mathbf{A}$ is symmetric and

$$
\mathbf{y}_{j}=\mathbf{A p}_{j} t_{j}, \quad j \geq 1
$$

it follows that

$$
\left(\mathrm{y}_{2}{ }^{T} \mathrm{H}_{2}-t_{2} \mathrm{p}_{2}{ }^{T}\right) \mathrm{y}_{1}=0
$$

So that Eqs. (6.3) are valid for $r=2$, and thus for all $r, 1 \leq r \leq n$. The theorem then follows by appeal to Theorem 2 and its corollary.

Theorem 7. Algorithm 2 is exact if $\mathbf{A}$ is symmetric and nonsingular.

Proof. Again the proof is inductive. Let

$$
\begin{array}{ll}
\mathbf{q}_{i}^{T} \mathbf{y}_{i}=\mathbf{z}_{i}^{T} \mathbf{y}_{i}=1, & 1 \leq i \leq r, \\
\mathbf{q}_{i}^{T} \mathbf{y}_{j}=\mathbf{z}_{i}^{T} \mathbf{y}_{j}=0, & 1 \leq j<i \leq r,
\end{array}
$$




$$
\mathbf{H}_{i+1}=\mathbf{H}_{i+1}^{T}, \quad 1 \leq i \leq r .
$$

Now $t_{j}, 1 \leq j \leq r$, is chosen specifically to minimise $F$ and since $\mathbf{f}=\operatorname{grad} F$ this implies that $\mathbf{f}_{j+1}$, the value of $\mathbf{f}$ at the point where $F$ is minimal, is orthogonal to the step vector $\mathbf{p}_{j}$. Thus

$$
\mathbf{f}_{j+1}^{T} \mathbf{p}_{j} t_{j}=0, \quad 1 \leq j \leq r,
$$

and this becomes, from (2.6), (1.3) and the postulated symmetry of $\mathbf{H}_{j+1}$,

$$
\mathbf{p}_{j+1}^{T} \mathbf{y}_{j}=0, \quad 1 \leq j \leq r .
$$

It then follows from (6.7) and the corollary to Theorem 4 that

$$
\mathbf{p}_{r+1}^{T} \mathbf{Y}_{r}=\mathbf{0}
$$

and, since Eq. (6.4) is also valid,

$$
\mathbf{y}_{r+1}^{T} \mathbf{H}_{r+1} \mathbf{Y}_{r}=\mathbf{0} .
$$

Thus from Eqs. (4.11d), (6.8) and (6.9), if $\mathbf{q}_{r+1}$ and $\mathbf{z}_{r+1}$ are given by (6.2) then Eqs. (6.6) are valid with $r$ replaced by $r+1$. Since, if $q_{1}$ and $z_{1}$ are given by $(6.2)$ Eq. (6.5) is also valid, it follows from (6.7) that provided $\mathbf{H}_{1}$ is symmetric, (6.6) is valid for $r=2$ and hence for all $r, 1 \leq r \leq n$. The theorem then follows from Theorem 2 and its corollary.

Theorem 8. Algorithm 2 is stable when used to minimise a convex function provided that $\mathbf{H}_{1}$ is positive definite and $\beta_{i} \geq 0$.

Proof. The only denominators occurring in (6.2) are $\mathbf{y}_{i}{ }^{T} \mathbf{H}_{i} \mathbf{y}_{i}$ and $\mathbf{p}_{i}{ }^{T} \mathbf{y}_{i}$, and it follows from (4.11a, c) and (6.7) that

$$
\mathbf{p}_{i}{ }^{T} \mathbf{y}_{i}=\mathbf{f}_{i}{ }^{T} \mathbf{H}_{i} \mathbf{f}_{i} .
$$

Hence it is sufficient for stability that $\mathbf{H}_{i}$ is positive definite for all $i$ since in that case no denominator can become zero and no $\mathrm{H}_{i}$ can become singular. The proof is by induction. Assume that $\mathbf{H}_{i}$ is positive definite. Then some real nonsingular matrix $\mathbf{L}$ can be found such that

$$
\mathbf{L L}^{T}=\mathbf{H}_{i} \text {. }
$$

Define now $Q_{i+1}$ by

$$
Q_{i+1}=\mathbf{x}^{T} \mathbf{H}_{i+1} \mathbf{x}
$$

where $\mathbf{x}$ is an arbitrary nonzero vector, and define $\mathbf{u}, \mathbf{v}, \mathbf{w}$ by

$$
\mathbf{u}=\mathbf{L}^{T} \mathbf{f}_{i}, \quad \mathbf{v}=\mathbf{L}^{T} \mathbf{x}, \quad \mathbf{w}=\mathbf{L}^{T} \mathbf{y}_{i},
$$

where neither $\mathbf{u}, \mathbf{v}$ nor $\mathbf{w}$ is null. Then, from (6.10), it follows that

$$
\mathbf{p}_{i}^{T} \mathbf{y}_{i}=\mathbf{u}^{T} \mathbf{u}
$$

and hence, from (4.11d), (6.2) and (6.11),

$$
Q_{i+1}=\mathbf{v}^{T} \mathbf{v}-\frac{\left(\mathbf{v}^{T} \mathbf{w}\right)^{2}}{\mathbf{w}^{T} \mathbf{w}}+\frac{t_{i}\left(\mathbf{u}^{T} \mathbf{v}\right)^{2}}{\mathbf{u}^{T} \mathbf{u}}+\frac{\beta_{i} t_{i}}{\mathbf{u}^{T} \mathbf{u} \mathbf{w}^{T} \mathbf{w}}\left(\mathbf{u}^{T} \mathbf{u v} \mathbf{v}^{T} \mathbf{w}+\mathbf{w}^{T} \mathbf{w} \mathbf{v}^{T} \mathbf{u}\right)^{2}
$$

Denote now $F\left(\mathbf{x}_{i}+\mathbf{p}_{i} \Delta t\right)$ by $F_{i}+\Delta F$. Then, since $\mathbf{f}=\operatorname{grad} F$, for $\Delta t$ sufficiently small it follows that 


$$
\Delta F \simeq \mathbf{f}_{i}^{T} \mathbf{p}_{i} \Delta t
$$

and since $\mathbf{p}_{i}=-\mathbf{H}_{i} \mathbf{f}_{i}$ and $\mathbf{H}_{i}$ is positive definite by hypothesis $\Delta F$ will be negative, and $F$ reduced, for a positive $\Delta t$. It is thus always possible to choose $t_{i}>0$ when minimising the function during each iteration so that, if $\beta_{i}>0$, it follows that

$$
\left(\beta_{i} t_{i} / \mathbf{u}^{T} \mathbf{u} \mathbf{w}^{T} \mathbf{w}\right)\left(\mathbf{u}^{T} \mathbf{u} \mathbf{v}^{T} \mathbf{w}+\mathbf{w}^{T} \mathbf{w v}^{T} \mathbf{u}\right)^{2} \geq 0 .
$$

Thus, if

$$
Q_{i+1}>0, \quad \beta_{i}=0
$$

then, a fortiori, $Q_{i+1}>0, \beta_{i}>0$.

Now

$$
\mathbf{v}^{T} \mathbf{v}-\left(\mathbf{v}^{T} \mathbf{w}\right)^{2} / \mathbf{W}^{T} \mathbf{W} \geq 0
$$

by Cauchy's inequality, equality occurring only if $\mathbf{v}=\mathbf{w} k$, where $k$ is an arbitrary scalar multiplier. Furthermore, since $t_{i}>0$,

$$
t_{i}\left(\mathbf{u}^{T} \mathbf{v}\right)^{2} / \mathbf{u}^{T} \mathbf{u} \geq 0,
$$

equality occurring only if $\mathbf{u}$ and $\mathbf{v}$ are orthogonal. Thus if $\beta_{i}=0, Q_{i+1}=0$ only if $\mathbf{u}^{T} \mathbf{w}=0$. But

$$
\mathbf{u}^{T} \mathbf{W}=\mathbf{f}_{i}{ }^{T} \mathbf{H}_{i} \mathbf{y}_{i}
$$

and this becomes, from (4.11a), (6.12) and the symmetry of $\mathbf{H}_{i}$,

$$
\mathbf{u}^{T} \mathbf{w}=-\mathbf{u}^{T} \mathbf{u} \neq 0
$$

establishing (6.14). Thus if $\beta_{i} \geq 0, \mathbf{H}_{i+1}$ is positive definite if $\mathbf{H}_{i}$ is positive definite, and since $\mathbf{H}_{1}$ is positive definite by hypothesis, Theorem 8 follows.

The stability of Algorithm 1, about which no theorems are proved, depends on the choice of $t_{i}$ and if this is chosen to minimise $F$ the algorithm can be unstable. The algorithm has not been tested experimentally but as its exactness is independent of the choice of $t_{i}$ it is possible that, if a good criterion for choosing this parameter could be found, it might prove to be competitive. Neither has Algorithm 2 , in its most general form, been extensively tested. If $\beta_{i}=0$, however, the algorithm becomes the modified Davidon algorithm [4] and in this form has achieved considerable success.

Although the Davidon algorithm is theoretically stable, experiments carried out by the author indicate that this stability depends critically upon the accuracy to which each successive value of $t_{i}$ is obtained, and indeed for one problem, solved in eighteen iterations, $t_{i}$ was negative on twelve occasions, indicating some violation of the conditions on which Theorem 8 is based. The number of iterations required to obtain the solution also depends on the accuracy of $t_{i}$ and for the problem quoted varied between fourteen and twenty-three, the lower number corresponding to the least accurate determination of $t_{i}$. This wide variation of behaviour, which makes it difficult to determine the optimum tolerance to be imposed on $t_{i}$, makes comparison between different methods very risky and it would appear that a thorough experimental study of Algorithms 1 and 2 would be in the nature of a major undertaking. 
The author wishes to thank Mr. F. Ford of the English Electric Company Ltd., Whetstone, Leicester, for his comments on the first draft of this paper and to the Directors of the same Company, by whom the author was employed when this work was undertaken, for their permission to publish the results. He is also indebted to the referee for his suggestions, particularly that leading to a much improved proof of Theorem 1 .

Department of Statistics

University College of Wales

1. J. G. P. Barnes, "An algorithm for solving non-linear equations based on the secant method,” Comput. J., v. 8, 1965, pp. 66-72. MR 31 \#5330.

2. C. G. BROYDEN, "A class of methods for solving nonlinear simultaneous equations," Math. Comp., v. 19, 1965, pp. 577-593.

3. W. C. Davidon, Variable Metric Methods for Minimization, A.E.C. Research and Development Report ANL-5990 (Rev. TID-4500, 14th ed.), 1959.

4. R. Fletcher \& M. J. D. PowelL, "A rapidly convergent descent method for minimization," Comput. J., v. 6, 1963/1964, pp. 163-168. MR 27 \#2096.

5. A. M. OsTrowski, The Solution of Equations and Systems of Equations, Pure and Appl. Math., Vol. 9, Academic Press, New York, 1960. MR 23 \#B571.

6. Walter Rudin, Principles of Mathematical Analysis, 2nd ed., McGraw-Hill, New York, 1964. MR $29 \# 3587$.

7. J. Traub, Iterative Methods for the Solution of Equations, Prentice-Hall, Englewood Cliffs, N. J., 1964. MR $29 \# 6607$.

8. Philip Wolfe, "The secant method for solving nonlinear equations," Comm. Assoc. Comput. Mach., v. 2, 1959, pp. 12-13. 